\section{First month prednisone dose predicts prednisone burden during the following 11 months: an observational study from the RELES cohort}

G Ruiz-Irastorza, ${ }^{1} \mathrm{M}$ Garcia, ${ }^{1} \mathrm{G}$ Espinosa, ${ }^{2} \mathrm{~L}$ Caminal, ${ }^{3} \mathrm{~F}$ Mitjavila, ${ }^{4}$ R González-León, ${ }^{5}$ B Sopeña, ${ }^{6} \mathrm{~J}$ Canora, ${ }^{7} \mathrm{M}$ V Villalba, ${ }^{8} \mathrm{M}$ Rodríguez-Carballeira, ${ }^{9}$ J M López-Dupla, ${ }^{10} \mathrm{~J}$ L Callejas, ${ }^{11}$ A Castro, ${ }^{12} \mathrm{C}$ Tolosa, ${ }^{13}$ M E Sánchez-García, ${ }^{14}$ M Pérez-Conesa, ${ }^{15} \mathrm{~N}$ Navarrete-Navarrete, ${ }^{16}$ A P Rodríguez, ${ }^{17} \mathrm{M}$ T Herranz, ${ }^{18}$ L Pallarés, ${ }^{19}$ on behalf of RELES, Autoimmune Diseases Study Group (GEAS)
To cite: Ruiz-Irastorza G, Garcia M, Espinosa G, et al. First month prednisone dose predicts prednisone burden during the following 11 months: an observational study from the RELES cohort. Lupus Science \& Medicine 2016;3:e000153 doi:10.1136/lupus-2016000153

Received 3 March 2016 Revised 22 June 2016 Accepted 2 July 2016

\section{CrossMark}

For numbered affiliations see end of article.

Correspondence to Dr G Ruiz-Irastorza; r.irastorza@outlook.es

\section{ABSTRACT}

Aim: To study the influence of prednisone dose during the first month after systemic lupus erythematosus (SLE) diagnosis (prednisone-1) on glucocorticoid burden during the subsequent 11 months (prednisone-2-12). Methods: 223 patients from the Registro Español de Lupus Eritematoso Sistémico inception cohort were studied. The cumulative dose of prednisone- 1 and prednisone-2-12 were calculated and recoded into a four-level categorical variable: no prednisone, low dose (up to $7.5 \mathrm{mg} /$ day), medium dose (up to $30 \mathrm{mg} /$ day) and high dose (over $30 \mathrm{mg} /$ day). The association between the cumulative prednisone-1 and prednisone-212 doses was tested. We analysed whether the fourlevel prednisone-1 categorical variable was an independent predictor of an average dose $>7.5 \mathrm{mg} /$ day of prednisone-2-12. Adjusting variables included age, immunosuppressives, antimalarials, methyl-prednisolone pulses, lupus nephritis and baseline SLE Disease Activity Index (SLEDAI).

Results: Within the first month, 113 patients (51\%) did not receive any prednisone, 24 patients $(11 \%)$ received average low doses, 46 patients $(21 \%)$ received medium doses and 40 patients (18\%) received high doses. There was a strong association between prednisone- 1 and prednisone-2-12 dose categories $(p<0.001)$. The cumulative prednisone- 1 dose was directly associated with the cumulative prednisone-2-12 dose $(p<0.001)$. Compared with patients on no prednisone, patients taking medium (adjusted OR 5.27, 95\% Cl 2.18 to 12.73) or high-dose prednisone-1 (adjusted OR 10.5, 95\% Cl 3.8 to 29.17 ) were more likely to receive prednisone2-12 doses of $>7.5 \mathrm{mg} /$ day, while patients receiving lowdose prednisone- 1 were not (adjusted OR 1.4, 95\% Cl 0.0 .38 to 5.2 ). If the analysis was restricted to the 158 patients with a baseline SLEDAI of $\geq 6$, the model did not change.

Conclusion: The dose of prednisone during the first month after the diagnosis of SLE is an independent predictor of prednisone burden during the following 11 months.
Glucocorticoids constitute one of the main therapies for systemic lupus erythematosus (SLE). However, despite their proven efficacy, oral glucocorticoids are important predictors of irreversible damage in lupus. ${ }^{2}$ Therefore, new ways of treating lupus beyond the use of high-dose oral prednisone are needed.

Registro Español de Lupus Eritematoso Sistémico (RELES) is the first Spanish multicentric inception lupus cohort, a research project of the Spanish Group of Autoimmune Diseases within the Spanish Society of Internal Medicine. The analysis of patterns of initial therapy in 223 patients enrolled up to July 2014 has been recently published. ${ }^{3}$ Our database offers the opportunity of studying the influence of prednisone therapy during the first month after the diagnosis on the glucocorticoid burden during the subsequent 11 months of follow-up.

\section{METHODS}

Patients at the time of SLE diagnosis, defined as meeting at least four American College of Rheumatology classification criteria, ${ }^{4}$ were included since January 2009. A total of 32 Internal Medicine Departments from hospitals all over Spain participated in this study. All patients signed an informed consent document at the time of enrolment. The study protocol was approved by the Institutional Research Ethics Boards of the coordinating centre (Hospital Universitario Cruces) and of all participating centres.

Detailed characteristics of the database have been recently published. ${ }^{3}$ Relevant to this study, every modification of therapy was entered in the database, so that it was 
possible to calculate the cumulative dose of immunosuppressive and immunomodulatory drugs for a given period of time. For the purposes of this study, the cumulative dose of prednisone at the first month (prednisone-1) and within the following 11 months (prednisone-2-12) were calculated and transformed into average daily doses, expressed in milligram/day. Such continuous variables were further recoded into a four-level categorical variable according to the classification by Buttgereit $e t a l^{5}$ : no prednisone, low dose (up to $7.5 \mathrm{mg}$ /day), medium dose (up to $30 \mathrm{mg} /$ day) and high dose (over $30 \mathrm{mg} /$ day).

\section{Statistical analysis}

Descriptive data were generated, using percentages, means and SDs. This study aimed to find associations between prednisone-1 and prednisone-2-12. The association between the four-level categorical prednisone-1 and prednisone-2-12 variables (no prednisone, low, medium and high doses) was tested by McNemar's test. The association between the cumulative prednisone-1 and prednisone-2-12 doses was tested by linear regression, adjusted by the use of immunosuppressive drugs, weeks on antimalarials, methyl-prednisolone pulses, presence of nephritis and baseline SLE Disease Activity Index (SLEDAI). We also analysed whether the four-level prednisone-1 categorical variable was an independent predictor of an average dose $>7.5 \mathrm{mg} /$ day of prednisone 2-12. This cut-off point was selected according to the published data identifying this dose as the limit for glucocorticoid-related damage accrual. ${ }^{6}$ Logistic regression was used for the analysis, with age at onset, immunosuppressive drugs, weeks on antimalarials, methyl-prednisolone pulses, lupus nephritis and baseline SLEDAI included as independent covariables. In order to eliminate the bias caused by mild disease, the analysis was repeated after excluding patients with a baseline SLEDAI of $<6$. All the statistical calculations were made with STATA (V.11.2; STATA, Texas, USA).

\section{RESULTS}

A total of 223 patients were included in this study. The main clinical characteristics of the cohort are shown on table 1 . About $89 \%$ of patients were women, $86 \%$ were Caucasians and $21 \%$ presented with lupus nephritis. The mean (SD) SLEDAI score at diagnosis was 9.8 (7.8). Of note, 158 patients $(70 \%)$ had a baseline SLEDAI of $\geq 6$. By the end of the first year, the mean (SD) SLEDAI score had significantly decreased to 4.4 (4.3), $\mathrm{p}<0.001$.

Fifty patients $(22 \%)$ had accrued any damage by the end of the first year of follow-up. During this period, no patients suffered avascular bone necrosis; three patients suffered vertebral crush fractures, aged 61, 23 and 75 years and having received average prednisone-2-12 doses of $1.8,18$ and $19.6 \mathrm{mg} /$ day, respectively.

Within the first month, 113 patients $(51 \%)$ did not receive any prednisone, 24 patients $(11 \%)$ received
Table 1 Baseline clinical manifestations in and treatments received within the first year by 223 patients of the Registro Español de Lupus Eritematoso Sistémico (RELES) cohort

\begin{tabular}{|c|c|}
\hline & $\mathbf{N}(\%)$ \\
\hline \multicolumn{2}{|l|}{ Clinical manifestations } \\
\hline Malar rash & $55(25 \%)$ \\
\hline Discoid rash & $17(8 \%)$ \\
\hline Subacute cutaneous lupus & $29(13 \%)$ \\
\hline Cutaneous vasculitis & $8(4 \%)$ \\
\hline Arthritis & $98(44 \%)$ \\
\hline Lupus nephritis & $47(21 \%)$ \\
\hline Class I & $2(1 \%)$ \\
\hline Class II & $3(1.5 \%)$ \\
\hline Class III & $5(2 \%)$ \\
\hline Class IV & $24(11 \%)$ \\
\hline Class V & $6(3 \%)$ \\
\hline No biopsy & 7 (4\%) \\
\hline Pleuritis & $28(13 \%)$ \\
\hline Pericarditis & $25(11 \%)$ \\
\hline Haemolytic anaemia & $19(9 \%)$ \\
\hline Thrombocytopenia & $32(14 \%)$ \\
\hline Venous thromboembolism & $22(10 \%)$ \\
\hline Stroke & $5(2 \%)$ \\
\hline \multicolumn{2}{|l|}{ Treatments } \\
\hline Prednisone & $144(65 \%)$ \\
\hline $\mathrm{HCQ}$ & $182(81 \%)$ \\
\hline Pulse methyl-prednisolone & $34(15 \%)$ \\
\hline Any immunosuppressive drug & $88(39 \%)$ \\
\hline Cyclophosphamide & $26(12 \%)$ \\
\hline Azathioprine & $32(14 \%)$ \\
\hline Methotrexate & $20(9 \%)$ \\
\hline Mycophenolate (sodium or mofetil) & $42(19 \%)$ \\
\hline Tacrolimus & $1(0.5 \%)$ \\
\hline
\end{tabular}

average low doses, 46 patients $(21 \%)$ received medium doses and 40 patients (18\%) received high doses (table 2). The use of medium-high doses of prednisone was associated with the presence of nephritis (OR 4.1, 95\% CI 1.9 to 8.9 ), thrombocytopenia (OR $4.3,95 \%$ CI 1.7 to 11.3 ), anti-DNA antibodies (OR 2.1, 95\% CI 1.07 to 4.3) and SLEDAI score at diagnosis of $\geq 6$ (OR 2.8, 95\% CI 1.3 to 6.1 ).

Table 2 Association between prednisone doses received during the first and subsequent 11 months of follow-up after the diagnosis of systemic lupus erythematosus

\begin{tabular}{|c|c|c|c|c|}
\hline \multirow[b]{2}{*}{ Prednisone-1 } & \multicolumn{4}{|c|}{ Prednisone-2-12 } \\
\hline & No & Low & Medium & High \\
\hline No $(n=113)$ & 79 (70\%) & $20(18 \%)$ & $14(12 \%)$ & $0(0 \%)$ \\
\hline Low $(n=24)$ & $1(4 \%)$ & $19(79 \%)$ & $4(17 \%)$ & $0(0 \%)$ \\
\hline $\begin{array}{l}\text { Medium } \\
(n=46)\end{array}$ & $2(4 \%)$ & $19(41 \%)$ & $25(54 \%)$ & $0(0 \%)$ \\
\hline High $(n=40)$ & $0(0 \%)$ & $10(25 \%)$ & 25 (62\%) & $5(13 \%)$ \\
\hline
\end{tabular}

Prednisone-1: average prednisone dose during the first month. Prednisone-2-12: average prednisone dose during months 2-12. 
There was a strong association between prednisone-1 and prednisone-2-12 dose categories $(\mathrm{p}<0.001$, table 2$)$. Of note, $54 \%$ of patients treated with medium doses during the first month received medium average prednisone 2-12 doses; among those treated initially with high doses, the proportion of patients receiving average prednisone doses of $>7.5 \mathrm{mg}$ /day increased to $75 \%$.

Likewise, the cumulative prednisone-1 dose during the first month was directly associated with the cumulative prednisone-2-12 dose $\left(\mathrm{R}^{2} 0.3, \mathrm{p}<0.001\right)$. This association remained unchanged after adjusting for age, immunosuppressive drugs, antimalarials, methyl-prednisolone pulses, presence of nephritis and baseline SLEDAI $(p<0.001)$.

Patients taking medium or high doses of prednisone-1 were more likely to be treated with prednisone-2-12 doses of $>7.5 \mathrm{mg} /$ day, compared with patients on no prednisone (OR 8.4, 95\% CI 3.7 to 18.8 and OR 21.2, $95 \%$ CI 9.8 to 52.6, respectively); on the contrary, patients receiving low doses during the first month were not (OR 1.4, 95\% CI 0.87 to 4.7). After adjusting for immunosuppressive drugs, antimalarials, methylprednisolone pulses, presence of nephritis, age and baseline SLEDAI, the results were similar (table 3). If the analysis was restricted to the 158 patients with a baseline SLEDAI of $\geq 6$, the model did not change substantially (table 3 ). Of note, the use of immunosuppressive drugs predicted treatment with doses of prednisone of $>7.5 \mathrm{mg} /$ day; however, adding methyl-prednisolone pulses to the therapeutic scheme reduced the odds of being treated with medium-high doses of prednisone-212 , particularly in patients with a baseline SLEDAI of $\geq 6$ (table 3). On the other hand, antimalarials had no effect, a fact that could be attributed to the high proportion of patients taking hydroxychloroquine (182/223, $82 \%)$, equally distributed in patients with and without prednisone $(12 / 144,84 \%$ vs $61 / 79,77 \%$, respectively, $\mathrm{p}=0.2$ ).

\section{DISCUSSION}

Early therapy of SLE often relies on glucocorticoids. ${ }^{78}$ In fact, there are not better drugs to achieve a rapid effect in patients with active disease. However, prolonged treatment with prednisone is often devastating. Glucocorticoids therapy is a major predictor of irreversible damage in SLE, showing a strong relation with complications such as osteonecrosis, osteoporotic fractures, diabetes, cataracts or cardiovascular disease, ${ }^{16}$ not to mention the profound physical changes that can exert a long-lasting impact on the quality of life of young patients. Treat-to-target recommendations by an international task force aim for the lowest glucocorticoid dosage needed to control disease during lupus maintenance treatment. ${ }^{9}$ Recent data have shown that average doses of prednisone over $7.5 \mathrm{mg}$ /day during the first year of disease course significantly increase damage accrual within 5 years. ${ }^{6}$ Moreover, data showing similar efficacy of regimes based on combination therapy
Table 3 Adjusted logistic regression model for predictors of average doses of prednisone $>7.5 \mathrm{mg} /$ day during the months $2-12$ of follow-up after systemic lupus erythematosus (SLE) diagnosis, in the whole cohort and limited to patients with a baseline SLEDAI of $\geq 6$

\begin{tabular}{|c|c|c|}
\hline & OR & $95 \% \mathrm{Cl}$ \\
\hline \multicolumn{3}{|l|}{ Whole cohort $(n=223)$} \\
\hline \multicolumn{3}{|l|}{ Prednisone-1 } \\
\hline No & Reference & Reference \\
\hline Low & 1.43 & 0.38 to 5.3 \\
\hline Medium & 5.8 & 2.3 to 14.3 \\
\hline High & 10.8 & 3.9 to 29.8 \\
\hline $\begin{array}{l}\text { Methyl-prednisolone bolus } \\
\text { (yes/no) }\end{array}$ & 0.32 & 0.10 to 1.02 \\
\hline $\begin{array}{l}\text { Immunosuppressive drugs } \\
\text { (yes/no) }\end{array}$ & 2.88 & 1.21 to 6.8 \\
\hline Weeks on $\mathrm{HCQ}$ & 1.00 & 0.99 to 1.00 \\
\hline Baseline SLEDAI & 1.07 & 1.01 to 1.14 \\
\hline Nephritis & 1.67 & 0.53 to 5.1 \\
\hline Age at diagnosis & 0.98 & 0.96 to 1.01 \\
\hline \multicolumn{3}{|c|}{ Baseline SLEDAI of $\geq 6$ ( $n=158)$} \\
\hline \multicolumn{3}{|c|}{ Prednisone-1 } \\
\hline No & Reference & Reference \\
\hline Low & 1.49 & 0.29 to 7.5 \\
\hline Medium & 4.6 & 1.65 to 12.9 \\
\hline High & 11.4 & 3.5 to 37.03 \\
\hline $\begin{array}{l}\text { Methyl-prednisolone bolus } \\
\text { (yes/no) }\end{array}$ & 0.13 & 0.03 to 0.57 \\
\hline $\begin{array}{l}\text { Immunosuppressive drugs } \\
\text { (yes/no) }\end{array}$ & 2.39 & 0.85 to 6.6 \\
\hline Weeks on $\mathrm{HCQ}$ & 1.00 & 0.99 to 1.00 \\
\hline Baseline SLEDAI & 1.06 & 0.98 to 1.13 \\
\hline Nephritis & 3.64 & 0.96 to 13.8 \\
\hline Age at diagnosis & 0.98 & 0.95 to 1.01 \\
\hline
\end{tabular}

containing lower doses of prednisone have been recently published, both in patients with ${ }^{10-12}$ and without lupus nephritis. ${ }^{13}$ Thus, maintenance doses of $>7.5 \mathrm{mg} /$ day could and should be avoided.

A recent study of the RELES cohort including these same 223 patients has revealed that as many as $38 \%$ of the patients were treated with average medium-high doses of prednisone (ie, $>7.5 \mathrm{mg}$ /day) during the first year after the diagnosis. ${ }^{3}$ We hypothesised that patters of glucocorticoid use are potentially dependent on the treating physician and this fact could be well investigated in this observational multicentric cohort in which therapeutic protocols were not predefined at all.

Our results confirm that, regarding prednisone use, what physicians did during the first month after the diagnosis of SLE was predictive of what happened within the following 11 months. Patients treated with initial low doses of prednisone were likely to be kept on low doses during the following months. On the other hand, half of the patients on initial medium doses received subsequent medium-high doses, a proportion that increased to $75 \%$ among those starting on high doses. It could be 
argued that higher initial doses are related with more severe disease and, thus, with higher needs of subsequent intensive therapy. However, this is only a partial explanation, since the influence of initial glucocorticoid therapy was independent from the baseline SLEDAI score and the presence of lupus nephritis, also in those patients presenting with a SLEDAI of $\geq 6$. Similar to previous results from the RELES cohort, immunosuppressive use did not result in reduced doses ${ }^{3}$ of prednisone while intravenous methyl-prednisolone bolus were related with lower doses of oral prednisone, particularly in patients with more active disease at presentation.

The dependence of the therapeutic protocol on the responsible physician/hospital could be seen as a limitation to this study. However, this heterogeneity in therapeutic protocols is actually the key in showing that the patterns of glucocorticoid use are more doctor-dependent than based on disease severity. The inception cohort design, the diagnosis of all patients after 2009 and the detailed acquisition of data on drug therapy are also important strengths of this study that make its conclusions quite solid. Unfortunately, due to the limited follow-up of 1 year, the analysis of the relation between general or glucocorticoid-related damage accrual and prednisone or intravenous methyl-prednisolone was premature, according to previous studies. ${ }^{6} 1013$ This will be hopefully possible with longer observational periods of our cohort.

Thus, it can be concluded that every effort should be made to limit oral glucocorticoid use since the very beginning of SLE course. Methyl-prednisolone bolus may help achieve rapid remission while reducing the need for oral prednisone. ${ }^{1314}$

\section{Author affiliations}

${ }^{1}$ Autoimmune Diseases Research Unit, Department of Internal Medicine, BioCruces Health Research Institute, Hospital Universitario Cruces, University of the Basque Country, Barakaldo, Bizkaia, Spain

${ }^{2}$ Department of Autoimmune Diseases, Hospital Clinic, Barcelona, Spain ${ }^{3}$ Department of Internal Medicine, Hospital Universitario Central de Asturias, Oviedo, Asturias, Spain

${ }^{4}$ Autoimmune Diseases Unit, Department of Internal Medicine. Hospital Universitario de Bellvitge, L'Hospitalet de Llobregat, Barcelona, Spain

${ }^{5}$ Department of Internal Medicine, Hospital Universitario Virgen del Rocío, Sevilla, Spain

${ }^{6}$ Department of Internal Medicine, Complejo Hospitalario Universitario de Vigo, Pontevedra, Vigo, Spain

${ }^{7}$ Department of Internal Medicine, Hospital Universitario Fuenlabrada, Fuenlabrada, Madrid, Spain

${ }^{8}$ Department of Internal Medicine, Hospital General Universitario Gregorio Marañón, Madrid, Spain

${ }^{9}$ Department of Internal Medicine, Hospital Universitario Mutua de Terrasa, Barcelona, Spain

${ }^{10}$ Department of Internal Medicine, Hospital Universitario Joan XXIII, Tarragona, Spain

${ }^{11}$ Department of Internal Medicine, Hospital Universitario San Cecilio, Granada, Spain

${ }^{12}$ Department of Internal Medicine, Hospital Universitario Sant Joan de Reus, Reus, Tarragona, Spain

${ }^{13}$ Department of Internal Medicine, Corporació Sanitària Parc Taulí, Sabadell, Barcelona, Spain

${ }^{14}$ Department of Internal Medicine, Autoimmune Diseases Unit, Hospital Universitario Reina Sofía, Córdoba, Spain
${ }^{15}$ Department of Internal Medicine, Hospital Universitario Miguel Servet, Zaragoza, Spain

${ }^{16}$ Department of Internal Medicine, Hospital Universitario Virgen de las

Nieves, Granada, Spain

${ }^{17}$ Department of Internal Medicine, Complejo Hospitalario Universitario de Ourense, Orense, Spain

${ }^{18}$ Department of Internal Medicine, Hospital J.M. Morales Meseguer, Murcia, Spain

${ }^{19}$ Department of Internal Medicine, Hospital Universitario Son Espases, Palma de Mallorca, Islas Baleares, Spain

Acknowledgements The authors thank Mar Ajubita and all the members of the Registry Coordinating Centre, S \& H Medical Science Service, for their logistic and administrative support.

Collaborators Appendix Members of the RELES-GEAS Group: M Akasbi (Department of Internal Medicine, Hospital Universitario Infanta Leonor, Madrid, Spain), R Boldova (Department of Internal Medicine, Hospital Clínico Universitario Lozano Blesa, Zaragoza, Spain), I Cabezas-Rodríguez (Department of Internal Medicine, Hospital Universitario Central de Asturias, Oviedo, Asturias, Spain), JL Callejas (Department of Internal Medicine, Hospital Universitario San Cecilio, Granada, Spain), L Caminal (Department of Internal Medicine, Hospital Universitario Central de Asturias, Oviedo, Asturias, Spain), J Canora (Department of Internal Medicine, Hospital Universitario de Fuenlabrada, Fuenlabrada, Madrid, Spain), N Carrasco (Department of Autoimmune Diseases, Hospital Clinic, Barcelona, Spain), MJ Castillo (Department of Internal Medicine, Hospital Universitario Virgen del Rocío, Sevilla, Spain), A Castro (Department of Internal Medicine, Hospital Universitario Sant Joan de Reus, Reus, Tarragona, Spain), AJ Chamorro (Department of Internal Medicine, Complejo Hospitalario Universitario de Ourense, Orense, Spain), JG Erdozain (Department of Internal Medicine, Hospital de Mendaro, Mendaro, Guipuzcoa, Spain), G Espinosa (Department of Autoimmune Diseases, Hospital Clinic, Barcelona, Spain), $F$ Fernández-Monrás (Department of Internal Medicine, Hospital Universitari Sagrat Cor, Barcelona, Spain), G Fraile (Department of Internal Medicine, Hospital Universitario Ramón y Cajal, Madrid, Spain), EM Fonseca (Department of Internal Medicine, Hospital de Cabueñes, Gijón, Vigo, Spain), B Frutos (Department of Internal Medicine, Hospital Universitario de Fuenlabrada, Fuenlabrada, Madrid, Spain), M García (Autoimmune Diseases Research Unit, Department of Internal Medicine, BioCruces Health Research Institute, Hospital Universitario Cruces, University of the Basque Country, Barakaldo, Vizcaya, Spain), M García-Morales (Department of Internal Medicine, Hospital Universitario San Cecilio, Granada, Spain), Al García-Sánchez (Department of Internal Medicine, Hospital Universitario Infanta Leonor, Madrid, Spain), A Gil (Department of Internal Medicine, Hospital Universitario La Paz, Madrid, Spain), R González-León (Department of Internal Medicine, Hospital Universitario Virgen del Rocío, Sevilla, Spain), MT Herranz (Department of Internal Medicine. Hospital J.M. Morales Meseguer, Murcia, Spain), R Hurtado (Department of Internal Medicine, Hospital General Universitario de Elche, Elche, Alicante, Spain), JF Jiménez-Alonso (Department of Internal Medicine, Hospital Universitario Virgen de las Nieves, Granada, Spain), JM López-Dupla (Department of Internal Medicine, Hospital Universitario Joan XXIII, Tarragona, Spain), A Marín-Ballvé (Department of Internal Medicine, Hospital Clínico Universitario Lozano Blesa, Zaragoza, Spain), H Martín-Álvarez (Department of Internal Medicine, Fundación Hospital de Alcorcón, Alcorcón, Madrid, Spain), ML Micó (Department of Internal Medicine, Hospital Universitario y Politécnico La $\mathrm{Fe}$, Valencia, Spain), F Mitjavila (Autoimmune Diseases Unit, Department of Internal Medicine, Hospital Universitario de Bellvitge, L'Hospitalet de Llobregat, Barcelona, Spain), N Navarrete (Department of Internal Medicine, Hospital Universitario Virgen de las Nieves, Granada, Spain), E Núñez-Cuerda (Department of Internal Medicine, Hospital General Nuestra Señora del Prado, Talavera de la Reina, Toledo, Spain), L Pallarés (Department of Internal Medicine, Hospital Universitario Son Espases, Palma de Mallorca, Islas Baleares, Spain), S Parra (Department of Internal Medicine, Hospital Universitario Sant Joan de Reus, Reus, Tarragona, Spain), I Perales (Department of Internal Medicine, Hospital Universitario de Fuenlabrada, Fuenlabrada, Madrid, Spain), M Pérez-Conesa (Department of Internal Medicine, Hospital Universitario Miguel Servet, Zaragoza, Spain), B Pinilla 
(Department of Internal Medicine, Hospital General Universitario Gregorio Marañón, Madrid, Spain), AP Rodríguez (Department of Internal Medicine, Complejo Hospitalario Universitario de Ourense, Orense, Spain), M Rodríguez-Carballeira (Department of Internal Medicine, Hospital Universitario Mutua de Terrasa, Terrasa, Barcelona, Spain), G Ruiz-Irastorza (Autoimmune Diseases Research Unit, Department of Internal Medicine, BioCruces Health Research Institute. Hospital Universitario Cruces, University of the Basque Country, Barakaldo, Vizcaya, Spain), L Sáez (Department of Internal Medicine, Hospital Universitario Miguel Servet, Zaragoza, Spain), G Salvador (Department of Internal Medicine, Hospital de Manises, Manises, Valencia, Spain), E Sánchez-García (Autoimmune Diseases Unit, Department of Internal Medicine, Hospital Universitario Reina Sofía, Córdoba, Spain), G Serralta (Department of Internal Medicine, Hospital Infanta Sofía, San Sebastián de los Reyes, Madrid, Spain), B Sopeña (Department of Internal Medicine, Complejo Hospitalario Universitario de Vigo, Pontevedra, Vigo, Spain), C Tolosa (Department of Internal Medicine, Corporació Sanitària Parc Taulí, Sabadell, Barcelona, Spain), MV Villalba (Department of Internal Medicine, Hospital General Universitario Gregorio Marañón, Madrid, Spain).

Contributors All authors included in this paper have fulfilled the criteria of authorship.

Funding This study was supported by the Spanish Society of Internal Medicine.

Competing interests None declared.

Ethics approval CEIC Hospital Universitario Cruces.

Provenance and peer review Not commissioned; externally peer reviewed.

Open Access This is an Open Access article distributed in accordance with the Creative Commons Attribution Non Commercial (CC BY-NC 4.0) license, which permits others to distribute, remix, adapt, build upon this work noncommercially, and license their derivative works on different terms, provided the original work is properly cited and the use is non-commercial. See: http:// creativecommons.org/licenses/by-nc/4.0/

\section{REFERENCES}

1. Zonana-Nacach A, Barr SG, Magder LS, et al. Damage in systemic lupus erythematosus and its association with corticosteroids. Arthritis Rheum 2000;43:1801-8.
2. Bruce IN, O'Keeffe AG, Farewell V, et al. Factors associated with damage accrual in patients with systemic lupus erythematosus: results from the Systemic Lupus International Collaborating Clinics (SLICC) Inception Cohort. Ann Rheum Dis 2015;74 1706-13.

3. Ruiz-Irastorza G, Garcia M, Espinosa G, et al. Patterns of drug therapy in newly diagnosed Spanish patients with systemic lupus erythematosus. Clin Exp Rheumatol 2016;34:466-72.

4. Hochberg MC. Updating the American College of Rheumatology revised criteria for the classification of systemic lupus erythematosus. Arthritis Rheum 1997;40:1725.

5. Buttgereit $F$, Straub $\mathrm{RH}$, Wehling $\mathrm{M}$, et al. Glucocorticoids in the treatment of rheumatic diseases: an update on the mechanisms of action. Arthritis Rheum 2004;50:3408-17.

6. Ruiz-Arruza I, Ugarte A, Cabezas-Rodriguez I, et al. Glucocorticoids and irreversible damage in patients with systemic lupus erythematosus. Rheumatology (Oxford) 2014;53:1470-6.

7. Alarcón GS, Friedman AW, Straaton KV, et al. Systemic lupus erythematosus in three ethnic groups: III. A comparison of characteristics early in the natural history of the LUMINA cohort. LUpus in MInority populations: NAture vs. Nurture. Lupus 1999;8:197-209.

8. Parker B, Urowitz MB, Gladman DD, et al. Impact of early disease factors on metabolic syndrome in systemic lupus erythematosus: data from an international inception cohort. Ann Rheum Dis 2015;74:1530-6.

9. van Vollenhoven RF, Mosca M, Bertsias G, et al. Treat-to-target in systemic lupus erythematosus: recommendations from an international task force. Ann Rheum Dis 2014;73:958-67.

10. Ruiz-Irastorza G, Danza A, Perales I, et al. Prednisone in lupus nephritis: how much is enough? Autoimmun Rev 2014;13:206-14.

11. Fischer-Betz R, Chehab G, Sander O, et al. Renal outcome in patients with lupus nephritis using a steroid-free regimen of monthly intravenous cyclophosphamide: a prospective observational study. J Rheumatol 2012;39:2111-17.

12. Zeher M, Doria A, Lan J, et al. Efficacy and safety of enteric-coated mycophenolate sodium in combination with two glucocorticoid regimens for the treatment of active lupus nephritis. Lupus 2011;20:1484-93.

13. Ruiz-Arruza I, Barbosa C, Ugarte A, et al. Comparison of high versus low-medium prednisone doses for the treatment of systemic lupus erythematosus patients with high activity at diagnosis. Autoimmun Rev 2015;14:875-9.

14. Parker BJ, Bruce IN. High dose methylprednisolone therapy for the treatment of severe systemic lupus erythematosus. Lupus 2007;16:387-93. 